\title{
One-pill once-a-day HAART: a simplification strategy that improves adherence and quality of life of HIV-infected subjects
}

This article was published in the following Dove Press journal:

Patient Preference and Adherence

3 May 2010

Number of times this article has been viewed

\author{
Monica Airoldi ${ }^{1,6}$ \\ Mauro Zaccarelli ${ }^{2}$ \\ Luca Bisi ${ }^{3}$ \\ Teresa Bini ${ }^{4}$ \\ Andrea Antinori ${ }^{2}$ \\ Cristina Mussini ${ }^{3}$ \\ Francesca Bai ${ }^{4}$ \\ Giancarlo Orofino ${ }^{5}$ \\ Laura Sighinolfi ${ }^{7}$ \\ Andrea Gori ${ }^{6}$ \\ Fredy Suter \\ Franco Maggiolo' \\ 'Division of Infectious Diseases, \\ Ospedali Riuniti, Bergamo; ${ }^{2}$ Clinical \\ Department, INMI L Spallanzani, \\ Roma; ${ }^{3}$ Division of Infectious \\ Diseases, Università, Modena; \\ ${ }^{4}$ Division of Infectious Diseases, \\ Ospedale San Paolo, Milano; ${ }^{5}$ Division \\ of Infectious Diseases, Ospedale \\ Amedeo di Savoia, Torino; ${ }^{6}$ Division \\ of Infectious Diseases, Ospedale \\ San Gerardo, Monza; ${ }^{7}$ Division \\ of Infectious Diseases, Ospedale \\ Sant'Anna, Ferrara, Italy
}

Correspondence: Franco Maggiolo Divisione di Malattie Infettive, Ospedali Riuniti, Largo Barozzi I, 24I 28 Bergamo, Italy

Fax +39035 253382

Email franco31556@hotmail.com
Objective: The aim of the ADONE (ADherence to ONE pill) study was to verify the effect of a reduced number of pills on adherence and quality of life (QoL) in HIV-infected patients on highly active antiretroviral therapy (HAART).

Design: Prospective, multicenter, study.

Methods: Patients chronically treated with emtricitabine (FTC) + tenofovir (TDF) + efavirenz (EFV) or lamivudine (3TC) + TDF + EFV and with a HIV-RNA $<50$ copies $/ \mathrm{mL}$ were switched to the single-pill fixed-dose regimen (FDR) of FTC + TDF + EFV. Data were collected with SF-36 using visual analog scales. Results of the final (6 months) primary as-treated analysis are reported.

Results: 212 patients (77.4\% males) of mean age 45.8 years were enrolled; 202 completed the study. One month post switch to FDR the adherence rate increased significantly to $96.1 \%$ from a baseline value of $93.8 \%(P<0.01)$. The increase was steadily maintained throughout the study (96.2\% at 6 months). QoL improved over time from $68.8 \%$ to $72.7 \%(P=0.042)$ as well, and was significantly associated with the perception of health status, presence of adverse events (AEs) and number of reported AEs $(P<0.0001)$. QoL significantly influenced adherence $(P<0.0001)$. During FDR use the mean CD4 count increased from 556 to 605 cells $/ \mu \mathrm{L}(P<0.0001)$. At the end of follow-up $98 \%$ of patients maintained HIV-RNA level $<50$ copies $/ \mathrm{mL}$ and $100 \%<400$ copies $/ \mathrm{mL}$. Four patients stopped therapy because they were lost to follow-up and 6 because of AEs (insomnia/nervousness 4, allergy 1, difficulties swallowing pills 1).

Conclusion: By substituting a one-pill once-a-day HAART, we observed an improvement of both adherence and QoL while maintaining high virologic and immunologic efficacy. HAART simplicity is an added value that favors adherence and may improve long-term success.

Keywords: EFV/FTC/TDF, single-pill regimen, once-daily antiretroviral regimen, adherence, QoL, patient preferences

\section{Introduction}

Adherence is a well-known problem in medicine. Especially in chronic, nonsymptomatic diseases, keeping to the prescribed treatment has proven difficult for patients. ${ }^{1}$ For HIV-infected patients on highly active antiretroviral therapy (HAART), adherence is of outmost importance. Poor adherence may lead to treatment failure, selection of viral mutations and development of drug resistance. ${ }^{2,3}$ On the basis of earlier studies, an adherence level of $95 \%$ or more seems necessary to prevent virologic failure; ${ }^{4}$ more recent experience has shown that durable viral suppression can be achieved by using regimens requiring lower adherence rates ${ }^{5-7}$ and that the relationship between adherence and the development of resistance differs by drug class. ${ }^{8-10}$ 
Adherence can be considered an ever-changing and adapting process influenced by socio-economic, healthrelated, treatment-related, and behavior-related factors. ${ }^{11-13}$

In the past, several studies have shown how treatment simplification strategies could enhance patients' adherence to HAART..$^{14-18}$ Although it has been postulated that this type of intervention acts through an improvement of patients' quality of life (QoL), a few studies have specifically addressed the relationship between QoL and adherence. ${ }^{11,19,20}$

ADONE (ADherence to ONE pill) is a qualitative study mainly intended to evaluate patient-oriented variables. Qualitative studies focus on the meaning people give to situations or events and are helpful to elucidate the processes and the interactions of different factors playing a role in determining adherence rates. In this trial we evaluated how therapy simplification could affect adherence, QoL and subjective perception of health status and how these variables did influence one another.

\section{Materials and methods}

ADONE is multicenter, open-label, comparative, prospective study with a within-patient analysis. The study was not intended to verify the effect of a single drug but to study the advantages of a switch strategy in terms of patients' oriented endpoints. The trial was conducted in 6 reference Italian Centers between March 2008 and April 2009.

Consecutive patients on a stable HAART and with a HIV-RNA level below the detection limit of 50 copies $/ \mathrm{mL}$ could be enrolled. To be eligible, patients must have been on a HAART regimen based on the association of lamivudine/ emtricitabine + tenofovir (3TC/FTC + TDF) either as single molecules or as a fixed-dose combination (FDC) plus efavirenz (EFV). 3TC and FTC, due to their pharmacokinetic and pharmacodynamic characteristics, were considered interchangeable. ${ }^{21}$ All patients were switched to a new regimen containing exactly the same active substances (FTC + TDF + EFV) but all in a FDC single pill. Patients, therefore, did not change their therapy in terms of active molecules or doses of the same molecules, but simply reduced the daily number of pills in their regimen from 3 or 2 to 1.

The main objective of the study was to verify if simplification of the antiretroviral regimen, measured as the mere reduction of pill burden, might affect patients' adherence rate. The primary evaluation was based on an AT (as treated) approach assuming as the end-point the variation of adherence rate at the end of the follow-up period compared to the respective baseline value.
Secondary objectives of the study were to verify the effect of the simplification strategy on QoL, to verify patients' preferences, to verify how adherence and QoL could change over time, and to establish virologic and immunologic outcomes of the simplified regimen.

The secondary objectives of the study were mainly intended to be evaluated in a descriptive form. However, the variation of QoL and patients' preferences were linked to the variation of adherence and to the virologic outcome of therapy, and inferential analysis was performed on these relationships.

All patient-related variables were collected at the moment of treatment switch and 1,2, 4 and 6 months after the switch by means of a self-reported questionnaire based on a modified version of Medical Outcomes Study Short-Form Health Survey (SF-36). ${ }^{22}$ To collect data, we used a visual analog scale (VAS) based on a $100 \mathrm{~mm}$ horizontal line. A VAS is a sensitive instrument to collect patients' perception of descriptive terms widely used in medicine ${ }^{23}$ and validated for the assessment of adherence to $\mathrm{HAART}^{24}$ and for the study of QoL ${ }^{25}$ in HIV patients.

The instrument was non-judgmental and was explained and administered in a confidential manner by a trained registered nurse.

Adherence was investigated with 4 separate questions considering 2 different recall periods: last month and last week. For each period patients were asked to report the proportion of doses taken and the proportion of doses taken with respect to the daily timing ( \pm 2 hours). In both cases a VAS scale was used to collect data.

Analysis of QoL was based on 3 groups of questions exploring limitations to: everyday social and work activities induced by either emotional of physical status; how much time in the past 4 weeks the patients experienced positive (eg, feeling full of life, feeling happy) or negative (feeling nervous, feeling worn-out) situations; and how true or false were statements investigating the current attitude and future concerns about their health status (eg, I seem to get sick more easily than other people, I am as healthy as anyone I know, I expect my health to get worse). A final QoL summary score was computed taking into account responses to all these items. An higher value indicated a better QoL.

A further question exploring the current perception and judgment of their health status was asked.

The presence/occurrence and severity of subjective symptoms were addressed by asking patients to report and grade any symptom experienced in the last 4 weeks using a 20 -item list. The following symptom categories were addressed: gastrointestinal symptoms (bloating, pain in the stomach, 
nausea and vomiting, diarrhea or loose bowel movements); respiratory symptoms (dyspnea); neurological symptoms (peripheral neuropathy, dizziness, muscle or joint pain); psychological discomfort (change in mood, problems having sex, changes in sleep pattern, trouble remembering, changes of body appearance); constitutional symptoms (fever, headache, fatigue, loss of appetite, weight loss or wasting); and allergy symptoms (rash, skin and hair problems). Symptoms were analyzed individually and collectively.

Patients' preferences and judgment of HAART was investigated by asking patients to quantify their personal judgment of the new therapy compared to the previous one in relation to 4 items: tolerability, convenience, simplicity and potency. A value of $50 \%$ indicated indifference, higher values a preference for the FDR, while lower values were indicative of a preference for the previous HAART regimen.

At baseline and 1, 2, 4 and 6 months after switching HAART, patients were tested for the occurrence of adverse events, hematological and chemical laboratory tests were performed, and patients were also tested for routine surrogate markers of HIV infection (HIV-RNA and CD4 T-cell counts).

The study was powered to evaluate superiority of the simplified regimen as compared to the regimen at baseline. Assuming a baseline mean adherence rate of $93 \%$ with a standard deviation of $11 \%$, a sample size of 154 patients was calculated to have $80 \%$ power to detect a difference in adherence proportion of 0.025 (eg, baseline mean of 0.930 and a post-baseline mean of 0.955 ), assuming a standard deviation of difference of 0.110 , and using a paired t-test with a 0.05 two-sided significance level. Being the primary analysis based on as treated approach, the sample size was adjusted to 180 patients based on an anticipated drop-out rate of $15 \%$.

The primary analysis was based on a per protocol approach (AT). Patients were counted in this analysis if they completed the 6-month follow-up. A secondary analysis was performed according to the intention-to-treat non-completer $($ ITT NC) $=$ failure approach. In this case all enrolled patients were included but patients not continuing the defined program for any reason were considered as failures. Each patient served as his or her own control.

Descriptive results are presented as proportions, means, range or $95 \%$ confidence intervals (CI). Inferential statistics using either parametric or non-parametric tests were used, as appropriate. Chi-square or Fisher's exact test were used to analyze categorical variables; ANOVA and Student's t-test were used for continuous variables unless they are not normally distributed, in which case the Mann-Whitney U tests or Wilcoxon signed rank test were used. Analysis of variance was used to explore the effect of multiple variables on specific outcomes.

All cited $P$ values are 2 -sided. All analyses were performed with the SPSS statistical software package for Windows, version 13.0.

The study was approved by each site's institutional review board. Appropriate informed consent was obtained from each participant and the clinical research was conducted in accordance with guidelines for the protection of human subjects.

\section{Results}

Two-hundred and twelve patients were enrolled and 202 completed the study. The enrolled subjects had a mean age of 45.8 years (range $28-75$ years) and $77.4 \%$ of them were males. Most had acquired HIV through heterosexual contacts $(43.9 \%)$, while homosexual sex was a risk factor in $39.2 \%$ and intravenous drug use in $15.6 \%$. Other risk factors for transmission counted for the remaining $1.4 \%$ of cases. A previous diagnosis of AIDS, according to the US Centers for Disease Control and Prevention (CDC) 1993 revised classification system for HIV infection, ${ }^{26}$ was present in $21.7 \%$ of subjects. Patients had been receiving combination antiretroviral therapy for a mean of 5.7 years (range 0.2 to 13.5 years) and had been on their current HAART for a mean of 2.1 years (range $0.2-6.8$ years). Overall, the mean number of HAART regimens the patients were exposed to was 2.4 (range 1-13), but $47.1 \%$ of subjects were on their first therapeutic regimen. Baseline CD4 T-cell counts ranged from 30 to 1989 cells/ $\mu \mathrm{L}$ (mean 556 cells $/ \mu \mathrm{L}$ ) and in all cases plasma HIV-RNA was below the limit of detection of 50 copies/mL.

\section{Adherence}

As expected, baseline adherence rates were high in patients fully responding to their therapy. The number of doses taken in the last month or in the last week were close to $97 \%$ and these values declined between $93 \%$ and $94 \%$ when dose timing was included in the question. Nevertheless, the simple switch to the FDC induced a sharp increase in adherence levels (Figure 1) that after a month were invariably significantly $(P<0.001)$ higher. Over time the increase in adherence rates was maintained although partially reduced. At the end of the follow up the doses taken over the previous months increased by $1.1 \%$ $(P=0.01)$; the doses taken in the same period of time with respect to the dosing schedule increased by $2.3 \%(P=0.002)$; 


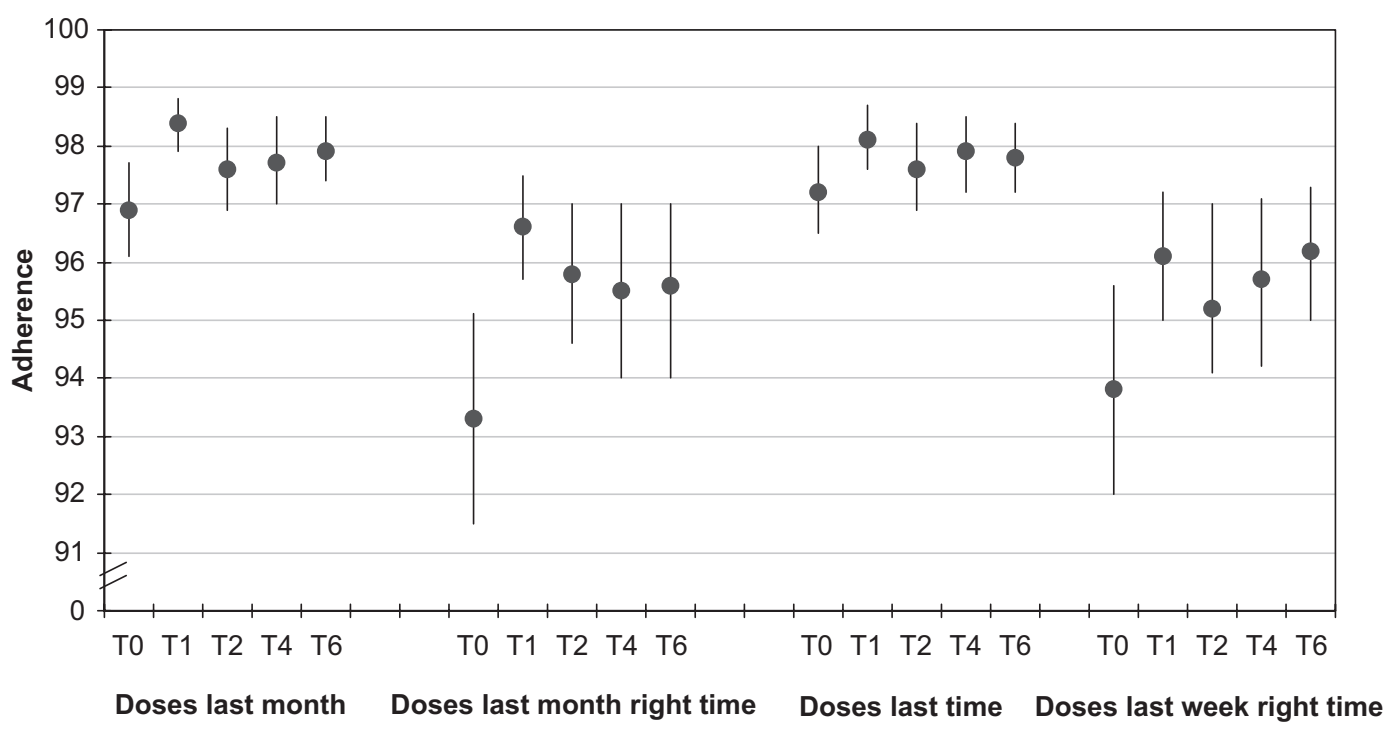

Figure I Self-reported adherence rates at different time points (T expressed in months) according to the specific recall periods and eventual dosing timing. Notes: The $Y$ axis represents the indication on the VAS scale and can be regarded as a percentage. Data are expressed as means and $95 \%$ confidence intervals.

those taken in the last week increased by $0.6 \%(P=0.116)$; and those taken in the last week with respect to the dosing timing increased by $2.4 \%(P=0.009)$ (Figure 1$)$.

\section{Perception of health status}

When actively asked, a relevant proportion of patients reported several symptoms that could be related to the taking of antiretroviral agents (Figure 2). Most patients (56.1\%) reported the presence of fatigue, while symptoms indicated by between $40 \%$ and $50 \%$ of patients included: muscles aches, sleep abnormalities, changes in body shape, diarrhea, problems with having sex, sadness or depression, nervousness or anxiety. Over time, the proportion of patients not reporting any symptoms significantly $(P<0.0001)$ improved, changing

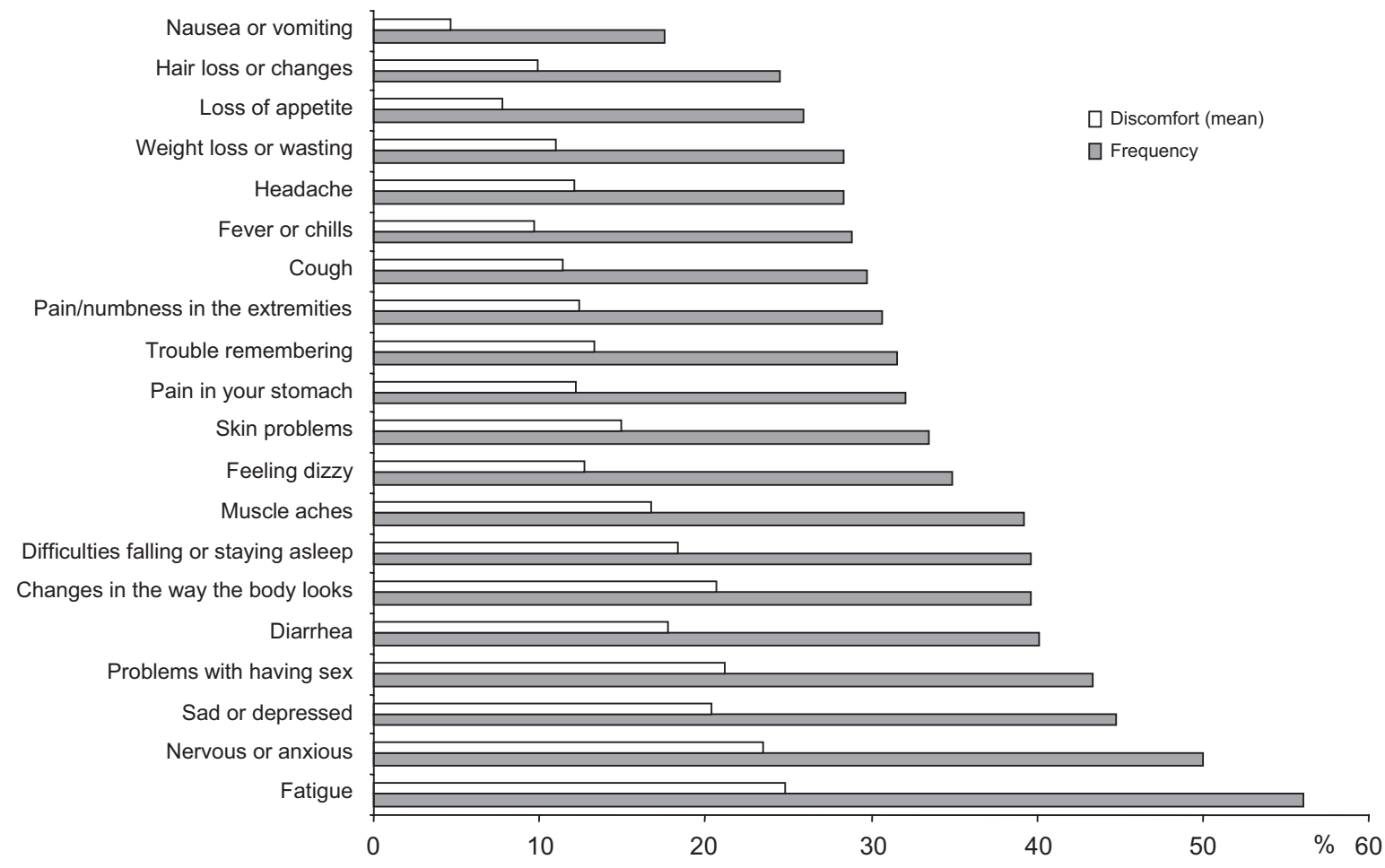

Figure 2 Baseline proportion of patients indicating the presence of specific symptoms possibly related to HAART. All symptoms were actively investigated by means of a structured questionnaire and patients were asked to grade the discomfort each symptom caused (VAS scale). 
from $9.9 \%$ (at baseline) to $30.7 \%, 6$ months after the switch to the FDC. The number of symptoms each patient reported significantly $(P=0.018)$ decreased, too. On the contrary, among patients reporting a given symptom, the perception of discomfort and the grading of it was rather stable over time. The presence and number of reported symptoms was strictly correlated $(P<0.0001)$ with the perception of health status by each patient (Figure 3 ) and how patients perceived their health status was a significant determinant of how subjects judged their limitations to everyday social and work activities; how much time in the past 4 weeks they experienced positive (eg, feeling full of life, feeling happy) or negative (feeling nervous, feeling worn-out) situations; and how true or false were statements investigating the current attitude and future concerns about their health status $(P<0.0001)$. Univariate analysis indicated that several other variables could influence perception of health status. The mean perception was lower in heterosexuals $(75.9 \%)$ and in intravenous drug users $(72.9 \%)$ and higher in men having sex with men $(81.9 \%)$ or in patients with other risk factors for HIV infec- tion $(92.6 \%)(P=0.003)$ and it was also correlated with the length of HAART $(P=0.031)$. However, when entered in a multivariate model, only the reported symptoms retained a strong statistical significance $(P<0.0001)$.

\section{Quality of life}

The various components of the QoL evaluation, although not always to a significant extent, invariably improved over time after the therapeutic switch. Limitations to everyday social and work activities declined from a baseline value of $17.3 \%$ (95\% CI 14.3 to 20.3 ) to $16.5 \%$ (95\% CI 13.7 to 19.3 ) after 6 months; the presence of positive feelings such as being full of life or happy increased from $67.8 \%$ of the time $(95 \%$ CI 64.3 to 71.4 ) at baseline to $70.2 \%$ (95\% CI 70.0 to 73.4 ) after 6 months, while time with negative feelings (nervous or worn-out) was reduced from $40.3 \%$ (95\% CI 37.0 to $43.6)$ to $31.5 \%$ (95\% CI 28.2 to 34.8$)(P<0.0001)$. Patients worried less about their current health status and were less concerned about the risk of it deteriorating in the future. On a scale with the most negative judgment equal to 100 and

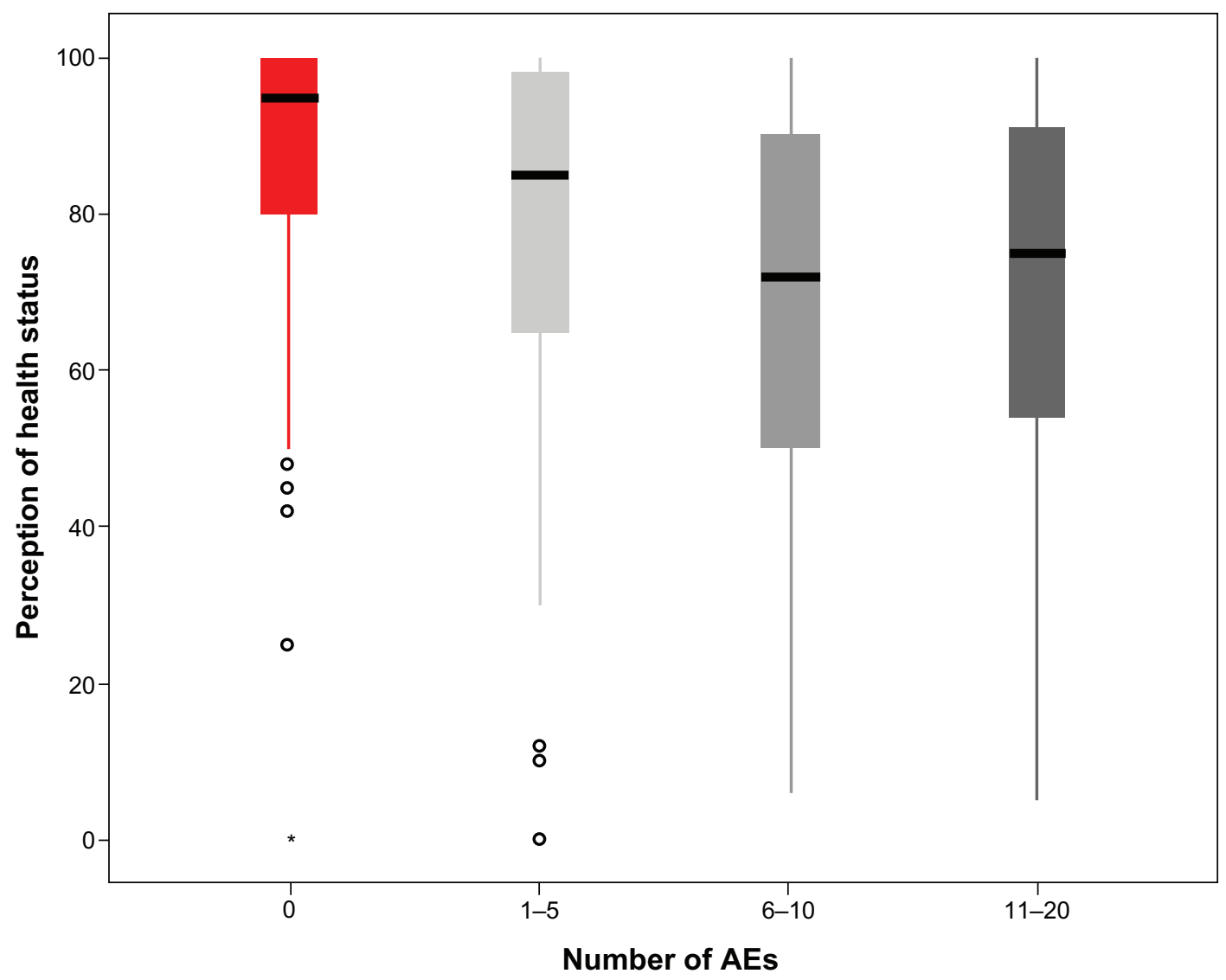

Figure 3 Influence of the presence and number of symptoms possibly related to HAART on the perception of health status at baseline. Box-plot analysis reporting median, interquartile range, $95 \% \mathrm{Cl}$ and outliers.

Abbreviation: AEs, adverse events. 
the best possible equal to 0 , their evaluation was $34.4(95 \%$ CI 31.3 to 37.5$)$ at baseline and 31.5 after 6 months (95\% CI 28.3 to 34.6). As a consequence the overall evaluation of QoL significantly $(P=0.042)$ increased over time from a baseline value of 68.8 (95\% CI from 67.7 to 70.0 ) to a value of 72.7 (95\% CI 71.5 to 73.8 ) after 6 months (Figure 4). QoL was strictly related to the perception of health status and to the presence of symptoms $(P<0.0001)$, too.

QoL levels significantly influenced adherence rates $(P<0.0001)$ (Figure 5). Patients in the lowest percentiles of QoL took 92.9\% of doses (95\% CI 83.4 to 100.0) in the last month; the doses taken in the same period of time with respect to the dosing schedule were $82.6 \%$ (95\% CI 66.0 to 99.1$)$; those taken in the last week were $94.8 \%$ (95\% CI 87.9 to 99.7$)$; and those taken in the last week with respect to the dosing timing were $85.2 \%$ (95\% CI 72.8 to 97.5 ); the equivalent figures in patients in the highest percentiles were 98.5 (95\% CI 98.3 to 99.1 ); $97.3 \%$ (95\% CI 92.9 to 96.4 ); 98.6\% (95\% CI 98.1 to 99.1 ); and $97.7 \%$ (95\% CI 96.2 to 99.2), respectively.

\section{Patients' preferences}

The comparative evaluation of the HAART regimen based on the use of single drug pills or on the FDC favored the last one in terms of patients' preferences concerning tolerability, convenience and simplicity. The preference was evident 1 month after the switch, but steadily increased over time (Figure 6) for all the considered characteristics of HAART. Of note, patients previously treated with lamivudine (thus receiving a regimen based on 3 pills) reported a higher preference rate for the FDC than those treated with emtricitabine (2 pills regimen because of the FDC emtricitabine/tenofovir). This difference was constant over time and expressed for all the characteristics, but was not statistically significant. However, the study was not powered to detect such a difference.

Interestingly, the patients' preference were in favor of the FDC in terms of efficacy, too (Figure 6).

\section{Immunologic response, virologic efficacy, tolerability}

Although the mean baseline CD4 count was fairly elevated in our patients, a steady increase of CD4 cells was observed over time (Figure 7, panel A). At any time point the intra-patients analysis yielded a statistically significant result $(P<0.001)$. After 6 months the mean CD4 increase was 49 cells $/ \mu \mathrm{L}$.

Virologic response to HAART was maintained throughout the study. At 6 months according to the AT approach, $100 \%$ of patients were below a HIV-RNA threshold of 400

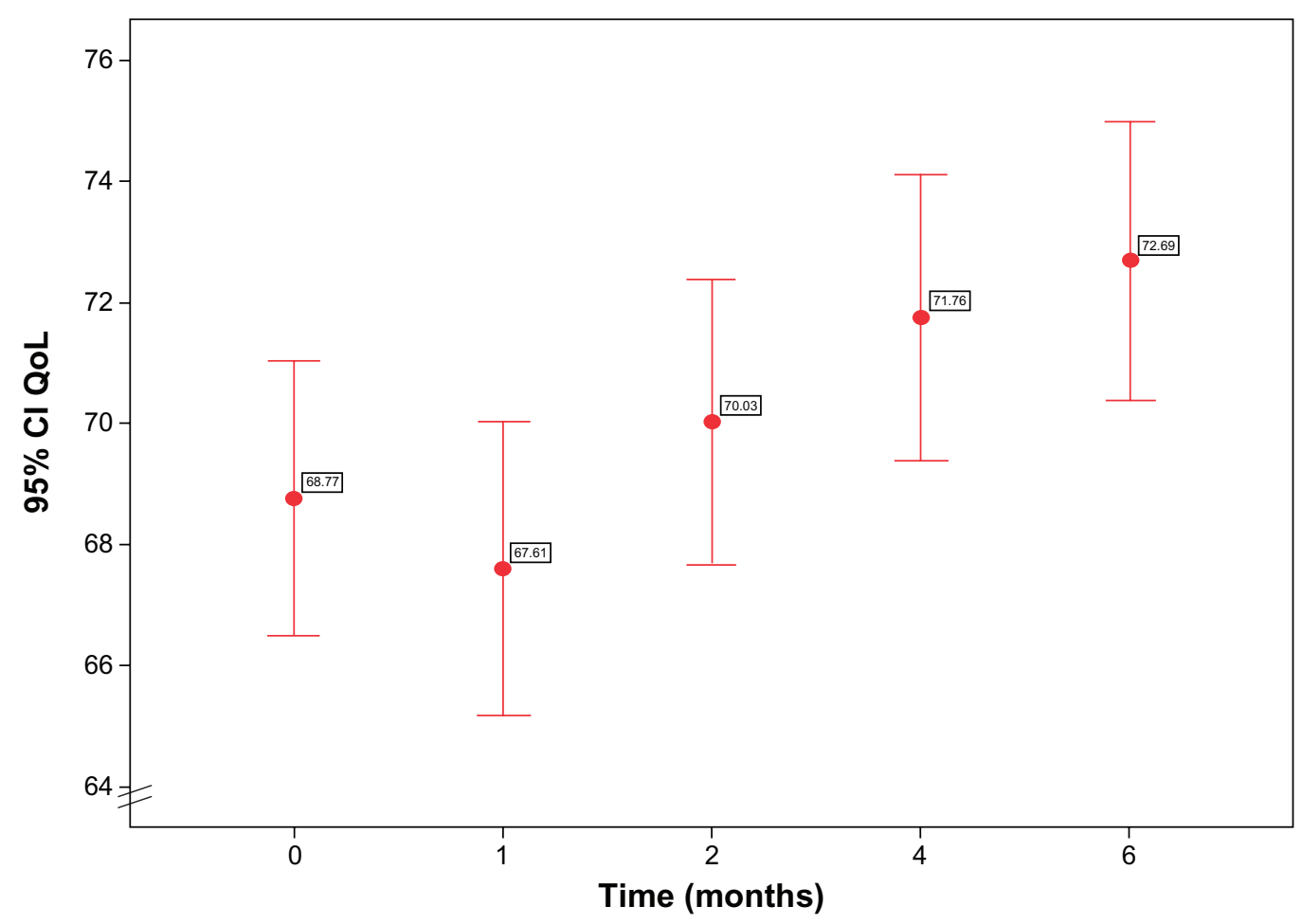

Figure 4 Variation of quality of life over time.

Note: Dots represent mean reported values and bars $95 \%$ confidence intervals.

Abbreviation: QoL, quality of life. 


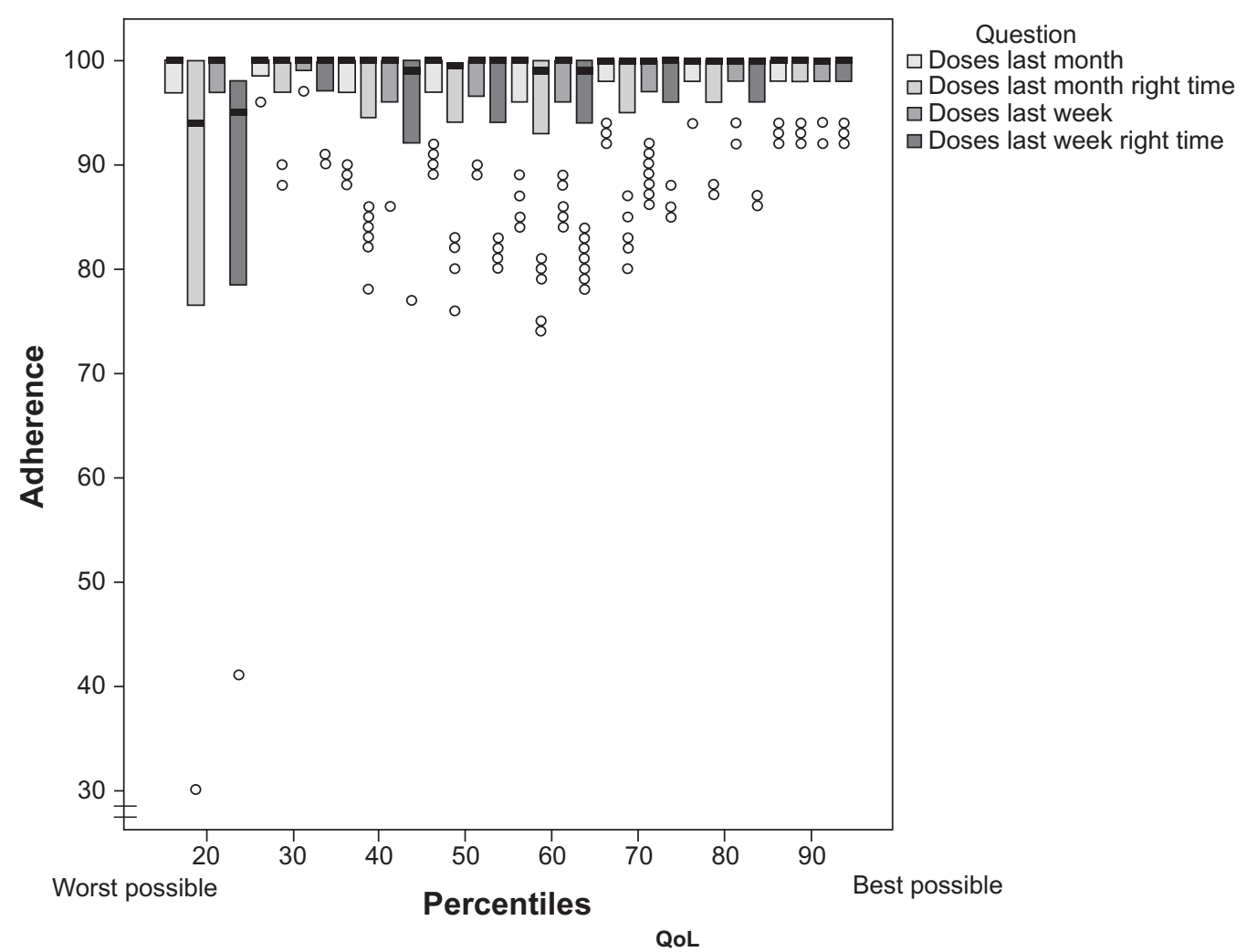

Figure 5 Influence of quality of life on adherence. Box-plot analysis reporting median, interquartile range, and outliers.

Abbreviation: QoL, quality of life.

copies/mL (Figure 7, panel B). Using a 50 copies $/ \mathrm{mL}$ limit, $98 \%$ of patients still presented a positive response. All the virologic rebounds we observed during the study period were transient viral blips of low intensity not present in successive HIV-RNA determinations. Furthermore adherence rates in patients presenting transient HIV-RNA elevations were even higher (doses taken in the last month $97.9 \%, 95 \%$ CI 96.2 to 99.7 ) than those of patients steadily maintaining viral suppression (97.6\%, 95\% CI 97.2 to 97.9). Similarly to these results, the ITT NC analysis revealed a positive response below 400 copies $/ \mathrm{mL}$ in $95.3 \%$ and below 50 copies $/ \mathrm{mL}$ in $93.4 \%$ of patients at 6 months.

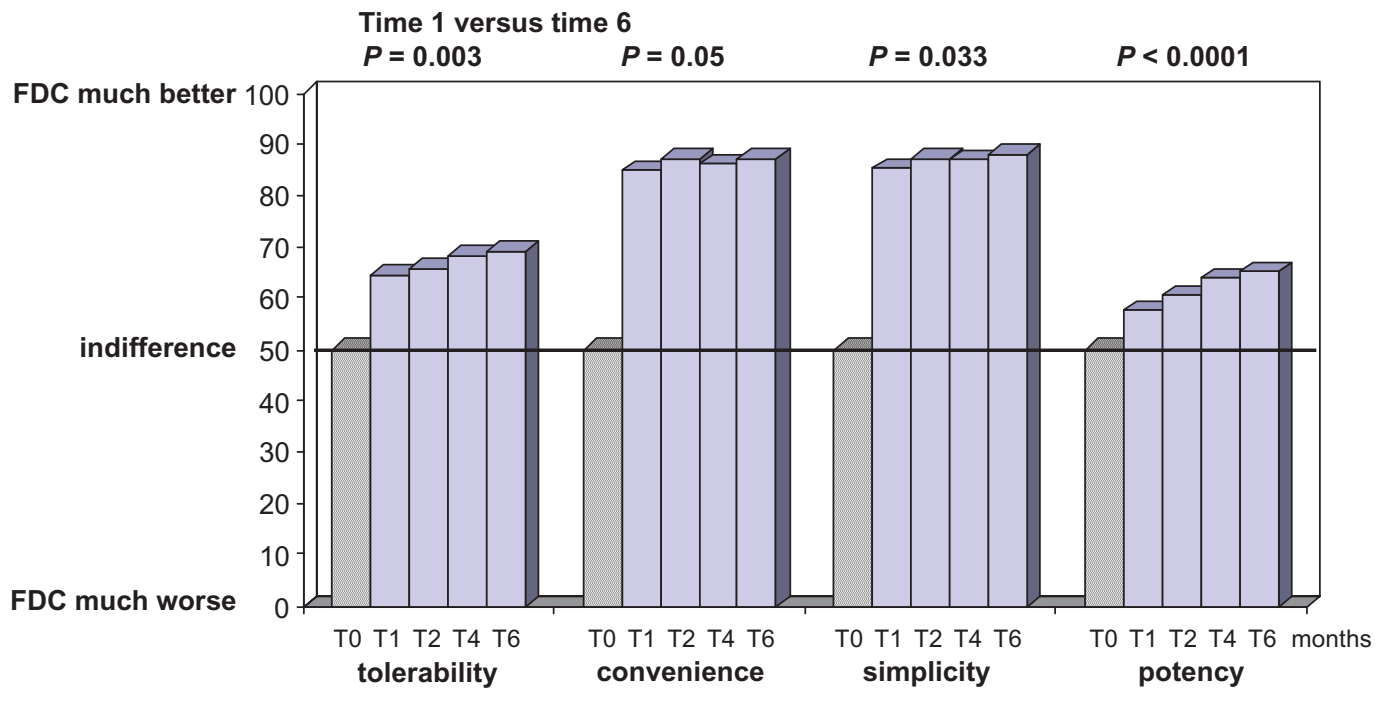

Figure 6 Patients' preferences. Patients' opinion was significantly in favor of the fixed dose combination (FDC) compared to the use of single drug pills (T0). Note: Statistics refers to differences observed during the FDC use (from I month versus 6 months after the switch). 


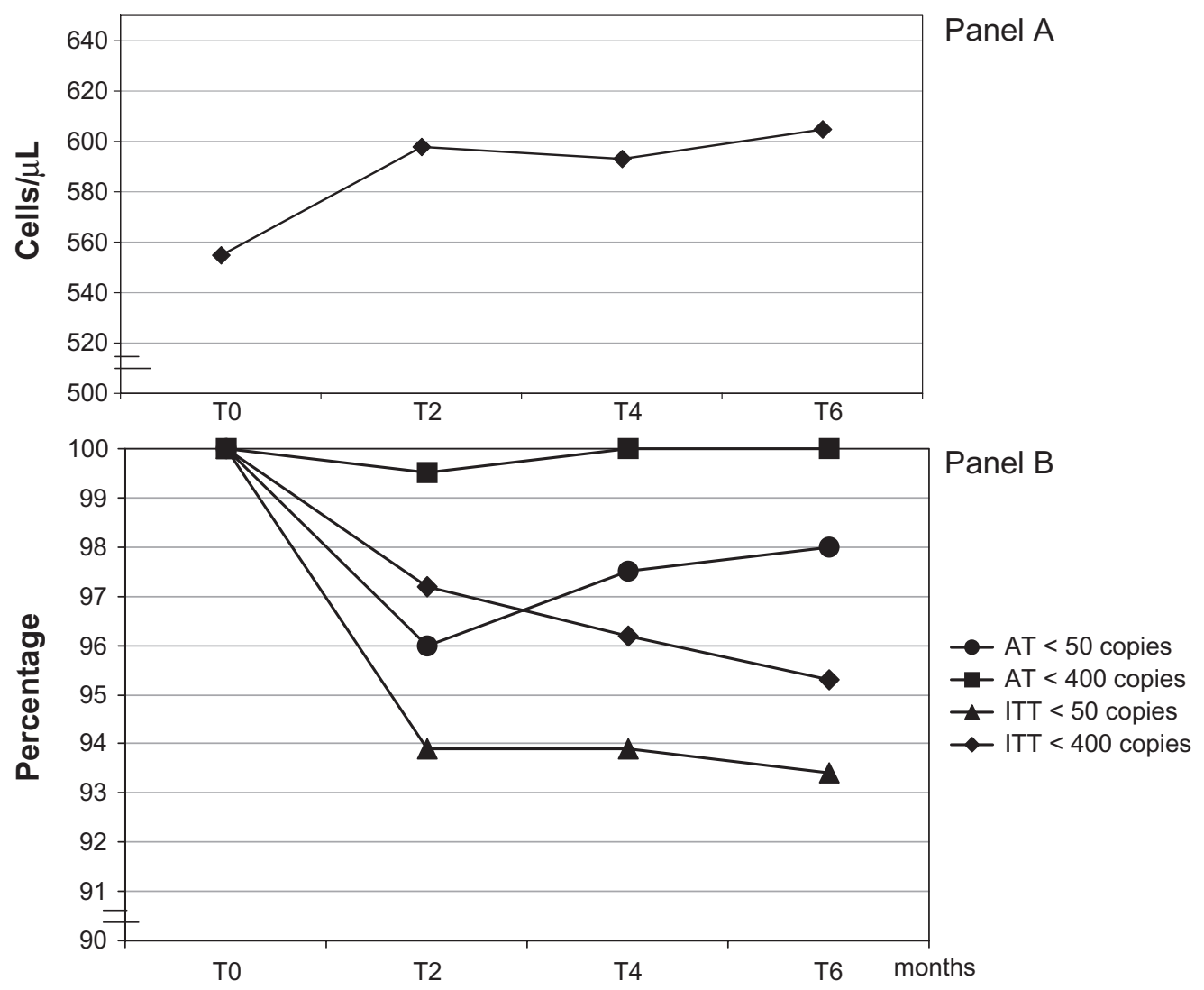

Figure 7 Immunolgic response (panel A) and virologic response (panel B). CD4 cell counts are expressed as means, while virologic outcome indicates the proportion of subjects below a determined threshold.

Six patients left the trial because of adverse events (AEs). In 4 cases CNS symptoms were involved (insomnia, altered dreams, nervousness), 1 patient presented a skin rash and 1 patient had difficulties in swallowing the pills. All of these re-started their previous HAART regimen without any further event. Four more patients stopped the trial because they moved to other centers.

\section{Discussion}

The complexity of medication regimens is often cited as one of the most important adherence barriers to HAART. Several surveys and cohort studies have shown that a number of regimen attributes, such as the number of daily pills, frequency and timing of doses, dietary restrictions, adverse events, medication storage requirements, are seen by patients as major factors making adherence difficult to the proposed HAART regimen. ${ }^{17,27}$ Several studies ${ }^{14-18}$ have explored how the reduction of pill burden and/or daily doses could affect adherence to antiretroviral regimens. Although the general conclusion was that simpler regimens were associated with better adherence rates, all these studies presented a common bias, as the compared therapies did not differ just for number of pills or doses, but were also based on different drugs. The confounding effect of different subjective tolerability could not, therefore, be ruled out. In this study, for the first time, the effect of simply reducing the number of pills in the regimen was evaluated in the absence of drug differences, assuming lamivudine equivalency (in tolerability terms) to emtricitabine. ${ }^{21}$ This approach was made possible by the use of a FDC pill combining all the drugs previously taken by our patients as separated entities.

Despite the fact that the study population comprised highly adherent patients, virologically responding to HAART, by simply reducing the number of pills of the therapeutic regimen, we obtained a significant increase in adherence that was maintained throughout the study period. It is known that self-reported adherence values may be higher, in absolute terms, than those derived with other measurement methods (ie, unnnounced pill counts or MEMS); ${ }^{9}$ however, because our data were comparable within the same patients and obtained by the same measurement method, their relative validity is maintained.

The observed increase at the end of follow-up was between $0.6 \%$ and $1.1 \%$ considering 1 -month or 1 -week 
recall, and between $2.3 \%$ and $2.4 \%$ considering dosing intervals. Although not large in absolute terms, these differences should be regarded as clinically meaningful, especially considering the direction of change. ${ }^{28}$ In fact, for adherence rates with respect to dose timing, the observed increases induced mean values always superior to the $95 \%$ (lower limit of $95 \%$ CI above 94\%) threshold, a limit often regarded as safe in terms of virologic efficacy of HAART., 4,29,30

A substantial proportion of subjects in our study reported adverse symptoms caused by, or suspected by patients to be a result of, antiretroviral therapy. The average discomfort/intensity caused by these symptoms, as reported by patients, was generally low and patients would not even have reported their occurrence to the care-givers if not actively questioned about it. However, it has been demonstrated that the presence of these symptoms is associated with intentional non-adherence ${ }^{31}$ and that patients' experience or fear of possible AEs is an important cause of reduced adherence. ${ }^{32}$ The presence and number of symptoms significantly affected perception of health status and, as already described, ${ }^{33}$ self-reported QoL, with QoL being inversely related to the number of symptoms. The reduction in the proportion of subjects reporting any subjective symptom and the reduction in the number of symptoms we observed after the switch to the FDR-based HAART paralleled the increased in selfreported QoL.

Perceived QoL was significantly associated with selfreported adherence, with patients in higher QoL percentiles showing better adherence rates. The difference was more pronounced when both the number and timing of doses concurred in defining the adherence rate. In this case being above the 50 percentile consistently assured adherence rates $>95 \%$. The increase in QoL observed after a therapeutic switch $(3.9 \%)$ could therefore play a relevant role in assuring better adherence and, as a consequence, long-term virologic efficacy of HAART.

The virologic efficacy of the FDR-based HAART was high. After 6 months of therapy $98 \%$ of patients still presented a HIV-RNA level $<50$ copies/mL (as treated population). Furthermore, all viral load increases observed were sporadic, of low intensity, and were not confirmed in successive samples. Because our patients were on an effective HAART for a long period of time, the release of virus from latently infected reactivated CD4 cells, rather than an ongoing viral replication, could be a likely explanation of these findings. ${ }^{34}$ This possible explanation is supported by the adherence rates reported by patients presenting viral blips, which did not differ and were even higher than those of patients with complete control of viral replication, and by the observation that $100 \%$ of patients on HAART presented a viral load $<400$ copies/mL. The high virologic efficacy observed while patients took the FDC-based HAART does not allow us to quantify the role of adherence on this outcome measure, but it is highly probable that enhanced adherence positively influenced this result.

The absence of a parallel control group does not allow us to quantify the role of switching therapy on the observed increase in CD4 T-cell counts. However, the increase was highly statistically significant and was observed at any time point of the follow-up period, indicating a constant positive effect of HAART on immunological response. This finding is in contrast with previously reported results with the FDCbased HAART. ${ }^{35}$

Four possible limitations to this study warrant specific mention. First, we were unable to define refusal rates, because data on potential participants to the study were not collected if patients were not interested. However, the demographic characteristics of enrolled patients are representative of the population of HIV-infected patients currently assuming HAART in Italy, providing some reassurance that our sample is representative of the population of interest. Second, trials that involve switching strategies tend to attract patients already motivated to make a change in treatment. As a consequence a possible intrinsic bias toward favoring the switch option under evaluation may be present. The motivation to simplify therapy in this stable population could have affected patients' reporting of investigated variables such as the occurrence of specific symptoms or adherence rates and/or medication preferences. However, over time, patients' consistency in reporting and grading AEs, adherence rates, QoL items, and therapeutic preferences was very high. An intentional reporting bias is therefore highly improbable, although psychological influences on the individual judgement cannot be excluded. Third, we chose an open-label design with intra-patient control (evaluating pre/post repeated measures with paired tests). Although this trial design has some limitations, its choice was based on several methodological considerations. The openlabel was the only possible option to evaluate the effect of pill reduction on outcome measures. The absence of a parallel control group did not allow for some inferential analysis, but did not introduce possible evaluation bias associated with higher drop-out rates in the control group ${ }^{35}$ determined by a desire to obtain the simpler regimen. Fourth, as the Italian health care system provides completely free-of-charge assistance and therapy to HIV-infected individuals, we did not include economic issues in the evaluation of health-related 
QoL. In other social settings where co-payments or private insurance fees are due, such variables could have a relevant effect on perceived QoL. ${ }^{36}$

Finally, when asked about regimen preferences, patients indicated the FDR-based HAART as highly preferable in terms of simplicity, convenience and tolerability. It is highly probable that patients' expectations and psychological motivations to simplify HAART did influence their judgment especially if we consider that the same preference was expressed in term of potency. However, it must be noted that patients previously treated with lamivudine and thus receiving a regimen based on 3 pills reported a higher preference rate for the FDC than those treated with emtricitabine who were on a previous HAART based on 2 pills because of the use of the emtricitabine/tenofovir combination. This difference was constant over time and was reported for all the investigated characteristics. Although not statistically significant (the study was not powered to detect such a difference) this observation clearly shows how even minimal simplifications are preferred by patients. In any case, we believe that patients' perception, rather than rational judgment, should be considered in evaluating these choices, as patients' perception may act as a trigger to make HAART more acceptable and to favor persistence on therapy.

In summary, this study confirms the high antiviral activity and tolerability of a single-pill HAART simplification strategy based on the use of a FDC containing emtricitabine, tenofovir and efavirenz. For the first time the crude effect of reducing the number of pills on adherence has been evaluated and results confirm that simpler regimens are associated with higher adherence rates, as well as improvement in QoL. Better adherence, high QoL and enhanced patients' preferences are all variables which might combine to assure long-lasting efficacy of HAART.

\section{Acknowledgments and disclosures}

The results presented in this paper were presented in part at the 9th International Congress on Drug Therapy in HIV Infection. 9-13 of November 2008, Glasgow, UK. Abstract P167.

We gratefully acknowledge the support of Gilead Sciences and Bristol-Myers Squibb who supplied the studied drugs.

\section{References}

1. Miller NH. Compliance with treatment regimens in chronic asymptomatic diseases. Am J Med. 1997;102:43-49.

2. Rodriguez-Rosado R, Jimenez-Nacher I, Soriano V, et al. Virological failure and adherence to antiretroviral therapy in HIV-infected patients. AIDS. 1998; 12:1112-1113.
3. Bangsberg DR, Charlebois ED, Grant RM, et al. High levels of adherence do not prevent accumulation of HIV drug resistance mutations. AIDS. 2003;17:1925-1932.

4. Paterson DL, Swindells S, Mohr J, et al. Adherence to protease inhibitor therapy and outcomes in patients with HIV infection. Ann Intern Med. 2000;133:21-30.

5. Maggiolo F, Ravasio L, Ripamonti D, et al. Similar adherence rates favour different virologic outcomes for patients treated with nonnucleoside analogues or protease inhibitors. Clin Infect Dis. 2005;40:158-163.

6. Bangsberg D. Less than $95 \%$ adherence to nonnucleoside reverse transcriptase inhibitor therapy can lead to viral suppression. Clin Infect Dis. 2006;43:939-941.

7. Shah B, Walshe L, Saple DG, et al. Adherence to antiretroviral therapy and virologic suppression among HIV-infected persons receiving care in private clinics in Mumbai, India. Clin Infect Dis. 2007;44:1235-1244.

8. Maggiolo F, Airoldi M, Kleinloog HD, et al. Effect of adherence to HAART on virologic outcome and on the selection of resistanceconferring mutations in NNRTI- or PI-treated patients. HIV Clin Trials. 2007;8:282-292.

9. Bangsberg DR, Acosta EP, Gupta R, et al. Adherence-resistance relationship for protease and non-nucleoside reverse transcriptase inhibitors explained by virological fitness. AIDS. 2006;20:223-231.

10. Bangsberg DR, Porco TC, Kagay C, et al. Modelling the HIV protease inhibitor adherence-resistance curve by use of empirically derived estimates. J Infect Dis. 2004;190:162-165.

11. Kleeberger CA, Phair JP, Strathdee SA, et al. Determinants of heterogeneous adherence to HIV-antiretroviral therapies in the multicenter AIDS Cohort Study. J Acquir Immune Defic Syndr. 2001;26:82-92.

12. Gordillo V, del Amo J, Soriano V, et al. Sociodemographic and psycological variables influencing adherence to antiretroviral therapy. J Acquir Immune Defic Syndr. 1999;13:1763-1769.

13. Vervoort SCJM, Borleffs JCC, Hoepelman AIM, et al. Adherence in antiretroviral therapy: a review of qualitative studies. AIDS. 2007;21:271-281.

14. Maggiolo F, Ripamonti D, Arici C, et al. Simpler regimens may enhance adherence to antiretrovirals in HIV infected patients. HIV Clin Trials. 2002;5:371-378.

15. Trotta MP, Ammassari A, Cozzi-Lepri A, et al. Adherence to highly active antiretroviral therapy is better in patients receiving non-nucleoside reverse transcriptase inhibitor-containing regimens than those receiving protease inhibitor-containing regimens. AIDS. 2003;17: 1099-1102.

16. Flandre P, Peytavin G, Meifreddy V, et al. Adherence to antiretroviral therapy and outcomes in HIV infected patients enrolled in an induction/ maintenance randomized trial. Antivir Ther. 2002;7:113-121.

17. Stone VE, Jordan J, Tolson J, et al. Perspectives on adherence and simplicity of HIV-infected patients on antiretroviral therapy. Self-report of the relative importance of multiple attributes of Highly Active Antiretroviral Therapy (HAART) regimens in predicting adherence. J Acquir Immune Defic Syndr. 2004;36:808-816.

18. Boyle B, Jayaweera D, Witt MD, et al. Randomization to once-daily stavudine extended release/lamivudine/efavirenz versus more frequent regimen improves adherence while maintaining viral suppression. HIV Clin Trials. 2008;9:164-176.

19. Singh N, Berman SM, Swindells S, et al. Adherence of human immunodeficiency virus-infected patients to antiretroviral therapy. Clin Infect Dis. 1999;29:824-830.

20. Holmes WC, Bilker WB, Wang H, et al. HIV/AIDS-specific quality of life and adherence to antiretroviral therapy over time. J Acquir Immune Defic Syndr. 2007;46:323-327.

21. Benson CA, van der Horst C, LaMarca A, et al. A randomized study of emtricitabine and lamivudine in stably suppressed patients with HIV. AIDS. 2004;18:2269-2276.

22. McHorney CA, Ware JE Jr, Raczek AE. The MOS 36-Item Short-Form Health Survey (SF-36) Psychometric and clinical tests of validity in measuring physical and mental health constructs. Med Care. 1993;31:247-263. 
23. Sriwatanakul K, Kelvie W, Lasagna L, et al. Studies with different types of visual analogue scales for measurement of pain. Clin Pharmacol Ther. 1983;34:234-239.

24. Giordano TP, Guzman D, Clark R, et al. Measuring adherence to antiretroviral therapy in a diverse population using a visual analogue scale. HIV Clin Trials. 2004;5:74-79.

25. Clotet B, Carmena J, Pulido F, et al. Adherence, quality of life, and general satisfaction with co-formulated zidovudine, lamivudine, and abacavir on antiretroviral-experienced patients. HIV Clin Trials. 2004; 5:33-39.

26. Centers for Disease Control and Prevention. 1993 revised system for HIV infection and expanded surveillance case definition for AIDS among adolescents and adults. MMWR. 1992;41:9-12.

27. Glass TR, De Geest S, Weber R, et al. Correlates of self-reported nonadherence to antiretroviral therapy in HIV-infected patients. The Swiss HIV Cohort study. J Acquir Immune Defic Syndr. 2006;41:385-392.

28. Hays RD, Woolley JM. The concept of clinically meaningful in healthrelated quality-of-life research. How meaningful is it? Pharmacoeconomics. 2000;18:419-423.

29. Bisson GP, Rowh A, Weinstein R, et al. Antiretroviral failure despite high levels of adherence: discordant adherence-response relationship in Botswana. J Acquir Immune Defic Syndr. 2008;49:107-110.

30. Golin CE, Earp J, Tien HC, et al. A 2-arm, randomized, controlled trial of a motivational interviewing-based intervention to improve adherence to antiretroviral therapy (ART) among patients failing or initiating ART. J Acquir Immune Defic Syndr. 2006;42:42-51.
31. Ammassari A, Murri R, Pezzotti P, et al. Self-reported symptoms and medication side effects influence adherence to highly active antiretroviral therapy in persons with HIV infection. J Acquir Immune Defic Syndr. 2001;28:445-449.

32. Heath KV, Singer J, O’Shaughnessy MV, et al. Intentional nonadherence due to adverse symptoms associated with antiretroviral therapy. J Acquir Immune Defic Syndr. 2002;31:211-217.

33. Mannheimer SB, Wold N, Gardner EM, et al. Mild-to moderate symptoms during the first year of antiretroviral therapy worsen quality of life in HIV-infected individuals. Clin Infect Dis. 2008;46:941-945.

34. Shen L, Peterson S, Sedaghat AR, et al. Dose-response curve slope sets class-specific limits on inhibitory potential of anti-HIV drugs. Nat Med. 2008; 14:762-766

35. DeJesus E, Young B, Morales-Ramirez JO, et al. Simplification of antiretroviral therapy to a single-tablet regimen consisting of efavirenz, emtricitabine and tenofovir disoproxil fumarate versus unmodified antiretroviral therapy in virologically suppressed HIV-1-infected patients. J Acquir Immune Defic Syndr. 2009;51:163-174.

36. Holmes WC, Bilker WB, Wang H, et al. HIV/AIDS-specific quality of life and adherence to antiretroviral therapy over time. J Acquir Immune Defic Syndr. 2007;46:323-327.
Patient Preference and Adherence

\section{Publish your work in this journal}

Patient Preference and Adherence is an international, peer-reviewed, open access journal that focusing on the growing importance of patient preference and adherence throughout the therapeutic continuum. Patient satisfaction, acceptability, quality of life, compliance, persistence and their role in developing new therapeutic modalities and compounds to

\section{Dovepress}

optimize clinical outcomes for existing disease states are major areas of interest. This journal has been accepted for indexing on PubMed Central. The manuscript management system is completely online and includes a very quick and fair peer-review system. Visit http://www.dovepress.com/ testimonials.php to read real quotes from published authors. 